\title{
Air coupled ultrasonic defect detection in polymer pipes
}

\author{
Arno Römmeler ${ }^{\mathrm{a}, \mathrm{b}, *}$, Roman Furrer ${ }^{\mathrm{a}}$, Urs Sennhauser ${ }^{\mathrm{a}}$, Bastian Lübke ${ }^{\mathrm{c}}$, Jörg Wermelinger $^{\mathrm{c}}$, Antonio de \\ Agostini $^{\mathrm{c}}$, Jürg Dual ${ }^{\mathrm{b}}$, Peter Zolliker ${ }^{\mathrm{a}}$, Jürg Neuenschwander ${ }^{\mathrm{a}}$ \\ ${ }^{a}$ Empa, Swiss Federal Laboratories for Materials Science and Technology, Dübendorf, Switzerland \\ ${ }^{b}$ ETH Zürich, Institute for Mechanical Systems, Zürich, Switzerland \\ ${ }^{c}$ Georg Fischer Piping Systems, Schaffhausen, Switzerland
}

\begin{abstract}
Ultrasonic, non-contact defect detection for polymer pipes can provide an easy and reliable method to establish on site quality control without destructive mechanical testing and finally may help to prevent damage from leaking pipes.

The target is to inspect thin walled polymer pipes made of PVDF and PP with small defects in the pipe wall. Air coupled transducers with a nominal frequency of $200 \mathrm{kHz}$ are used to excite Lamb waves in the pipe wall that interact with potential defects. A reference measurement on a flawless part of the pipe helps to mitigate the effects of geometrical and material inhomogeneities.

The possibility to excite only the $\mathrm{A}_{0}$ Lamb wave mode in thin plates and pipes using air coupled transducers is shown. Defects attenuate the propagating Lamb waves, resulting in a characteristic pattern in the receiver signal. Simulations on the Lamb wave propagation help to identify the dispersion parameters and allow to understand the effect of defects better. The detection of defects is challenging because of other imperfections in the specimen. With a reference measurement the detection of defects down to a size of $1 \mathrm{~mm}$ is possible. Keywords: air-coupled ultrasound, non-contact, guided waves, Lamb wave, polymer pipes
\end{abstract}

\section{Introduction}

Defect detection in pipes has a great potential to prevent leaks and severe damage. Ultrasonic inspection as a non destructive testing method allows pipe wall inspection locally and is a promising technique because of its high sensitivity for small cracks. Traditionally liquid coupled ultrasonic (LCUS) involves a coupling medium to guarantee a good connection between transmitter, material and receiver. Air coupled ultrasonics

\footnotetext{
* Corresponding author

Email address: arno.roemmeler@empa.ch (Arno Römmeler)
}

Preprint submitted to NDT and E International

December 14, 2018

This document is the accepted manuscript version of the following article:

Römmeler, A., Furrer, R., Sennhauser, U., Lübke, B., Wermelinger, J., de Agostini, A., ... Neuenschwander, J. (2018). Air coupled ultrasonic defect detection in polymer pipes. NDT and E International. https:// doi.org/10.1016/j.ndteint.2018.12.004 
(ACUS) inspection does not rely on any coupling agent and allows a contactless examination of pipes [1].

The application scenario of the air coupled ultrasonic inspection of pipes is defect detection, including cracks, production inhomogeneities as voids and the inspection of welds. All these defects have in common that they can only with huge effort be produced artificially. As a starting point, rather large through thickness holes were chosen to test the performance of this inspection method.

Because of the large difference of the acoustic impedance in air and polymers a large part of the emitted signal is reflected at the specimen surface and prevents a classical pulse-echo setup with a single probe. In thin walled structures as pipe walls it is possible to excite a Lamb wave with an air coupled transmitter. The Lamb wave propagates in direction of the long axis of the pipe, interacts with defects along the way and can be detected with an air coupled receiver. This single sided pitch-catch setup avoids the problem that the large signal from the surface reflection masks echos from defects inside the material.

Lamb waves were first described by Horace Lamb more than 100 years ago. The governing equations for anti-symmetric (1) and symmetric (2) Lamb wave modes are presented by Rose [2]:

$$
\begin{aligned}
& \frac{\tan (q h)}{\tan (p h)}=-\frac{\left(q^{2}-k^{2}\right)^{2}}{4 k^{2} p q} \\
& \frac{\tan (q h)}{\tan (p h)}=-\frac{4 k^{2} p q}{\left(q^{2}-k^{2}\right)^{2}}
\end{aligned}
$$

$$
p^{2}=\frac{\omega^{2}}{c_{1}^{2}}-k^{2}, q^{2}=\frac{\omega^{2}}{c_{2}^{2}}-k^{2}
$$

where $h$ is the plate thickness, $c_{1}$ and $c_{2}$ are the wave speeds in the bulk and therefore material parameters, $k$ is the wavenumber and $\omega$ is the angular frequency. From the numerical solutions of these equations the phase and group velocity for each propagating mode can be calculated. The Matlab Lamb toolbox [3] provides the possibility to calculate the dispersion relations from the bulk material parameters for the ideal case of a semi-infinite thin plate. Hartmann and Jarzynski and Christman characterized PVDF, PP and PMMA [4, 5]. While non contact testing provides a major advantage, the transition of the pressure wave into the structure is a big challenge. Anti-symmetric modes are easier to excite with a single-sided setup than symmetric modes. The efficiency of the coupling is depending on the transducer angle and on the excited 
Lamb mode. The sensitivity of the excitation of a specific Lamb mode with regards to the transducer angle

30 Leutenegger [6] investigated the non-destructive testing of metal tubes using a laser vibrometer. The air coupled technique in our setup allows a more compact setup and does not rely on reflective tape on the specimen surface, but lacks the information about the direction of the surface displacement.

The Lamb waves that propagate along the pipe wall interact with defects inside the wall. The waves are reflected or scattered by the defects or mode conversion can take place. Our transmission inspection setup can only detect the attenuation of the $\mathrm{A}_{0}$ Lamb wave that is caused by the defects. The scattering pattern for Lamb waves at delaminations is investigated by Veidt [7]. Hinders [8] and Fromme [9] investigated the scattering properties in plates of an incoming Lamb wave at through holes with sizes around $10 \mathrm{~mm}$. Using air coupled transducers with a setup very similar to ours, Padiyar [10] examined delaminations in the range 40 of $10-30 \mathrm{~mm}$ in composites.

Mode conversion at asymmetrical defects are investigated experimentally and theoretically by Benmeddour [11]. Ramadas [12] studied mode conversions of the incident $\mathrm{A}_{0}$ Lamb wave mode at delamination boundaries with air coupled transducers. Our setup does not allow to generate or detect $\mathrm{S}_{0}$ or higher Lamb modes, therefore the detection of defects using mode converted Lamb waves is not possible. Quaegebeur [13] investigated the quality control of cardboard tubes using air coupled ultrasonic inspection and reported the detection of thickness variations and a decrease of the Young's modulus in the pipe wall.

\section{Material and methods}

\subsection{Specimen}

In total six pipes were prepared for testing, three polyvinylidene difluoride (PVDF) and three polypropylene (PP) specimens with different diameters and wall thicknesses according to common standards as shown in Table 1. All pipe samples are $400 \mathrm{~mm}$ long and contain four through thickness holes as artificial defects in one cross sectional plane in the middle of the pipe. The sizes of the holes are $0.5 \mathrm{~mm}, 1 \mathrm{~mm}, 2 \mathrm{~mm}$ and $4 \mathrm{~mm}$ to include at least one hole that is almost impossible to detect and one hole that is detected with certainty. The holes are distributed along the circumference with $90^{\circ}$ spacing with respect to each other. 


\begin{tabular}{|l|l|r|r|}
\hline Material & Type & Diameter & Wall Thickness \\
\hline PP & Pipe & $110 \mathrm{~mm}$ & $10.4 \mathrm{~mm}$ \\
PP & Pipe & $63 \mathrm{~mm}$ & $6.0 \mathrm{~mm}$ \\
PP & Pipe & $40 \mathrm{~mm}$ & $3.8 \mathrm{~mm}$ \\
PVDF & Pipe & $110 \mathrm{~mm}$ & $3.7 \mathrm{~mm}$ \\
PVDF & Pipe & $63 \mathrm{~mm}$ & $3.2 \mathrm{~mm}$ \\
PVDF & Pipe & $40 \mathrm{~mm}$ & $2.6 \mathrm{~mm}$ \\
\hline
\end{tabular}

\begin{tabular}{|l|r|r|}
\hline Material & Type & Size $(\mathrm{L}, \mathrm{W}, \mathrm{Th})$ \\
\hline PMMA & Plate & $300 \times 100 \times 3 \mathrm{~mm}^{3}$ \\
\hline
\end{tabular}

Table 1: List of specimens and dimensions: three PP pipes, three PVDF pipes and one PMMA plate.

\begin{tabular}{|l|r|r|r|}
\hline Material & $\rho\left[\mathrm{kg} / \mathrm{m}^{3}\right]$ & $\mathrm{c}_{1}[\mathrm{~m} / \mathrm{s}]$ & $\mathrm{c}_{2}[\mathrm{~m} / \mathrm{s}]$ \\
\hline PP 4] & 913 & 2650 & 1300 \\
PVDF 4] & 1779 & 1930 & 775 \\
PMMA [5] & 1183 & 2746 & 1392 \\
Air & 1.195 & 343 & - \\
\hline
\end{tabular}

Table 2: Literature material parameters: density $(\rho)$ and the bulk wave speeds $\mathrm{c}_{1}$ and $\mathrm{c}_{2}$.

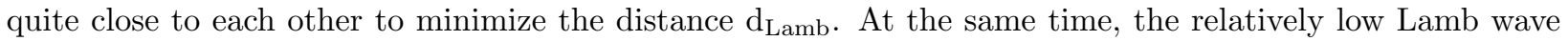
velocity in polymers in the range of two to three times the speed of sound in air gives only a small time frame in which the Lamb wave signal can be detected at the receiver before the direct signal through the air 


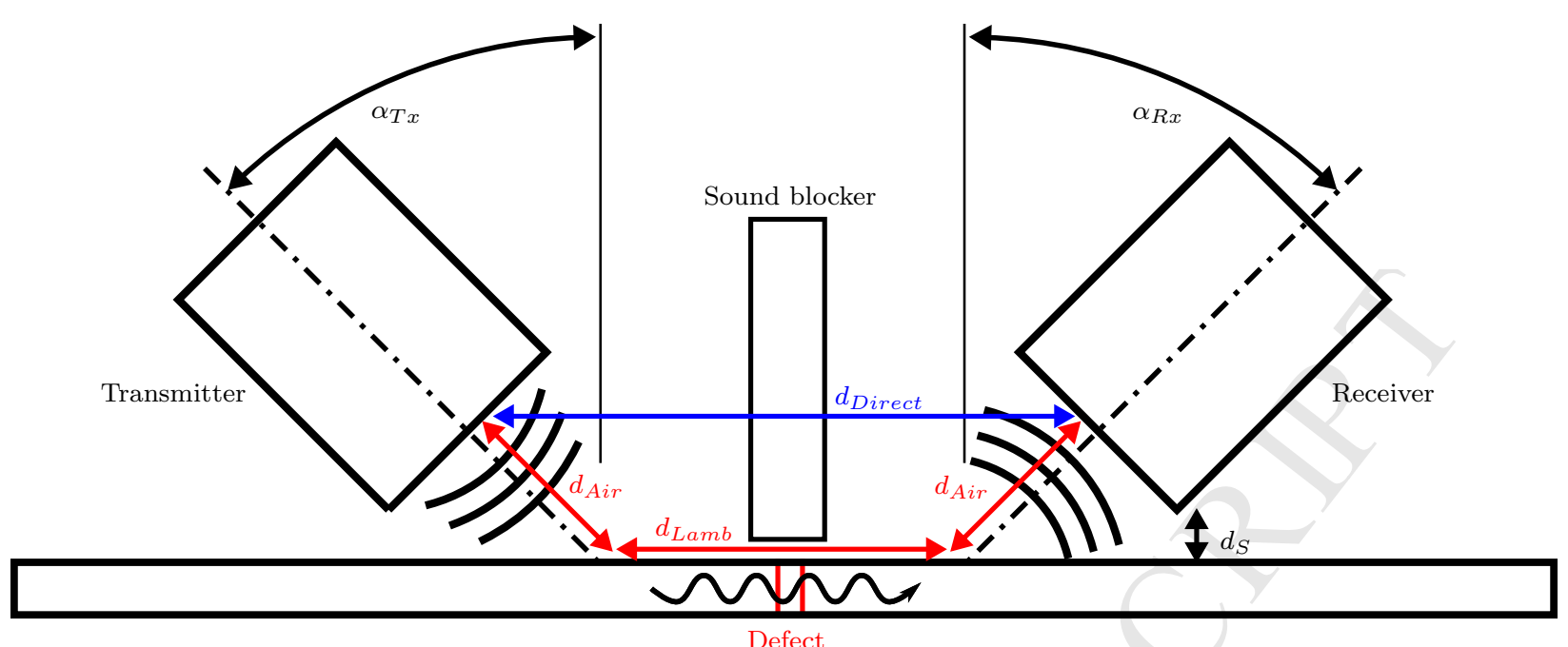

Figure 1: Setup for ACUS transmission inspection of thin walled structures. The inspection wave travels as pressure wave in air $\left(\mathrm{d}_{\text {air }}\right)$, as Lamb wave in the pipe wall $\left(\mathrm{d}_{\text {Lamb }}\right)$ where it interacts with possible defects and again as pressure wave back to the receiver $\left(\mathrm{d}_{\text {air }}\right)$. The transducer angles $\alpha_{T x}$ and $\alpha_{R x}$ can be adapted to the specific material requirements. An optional sound blocker is added to suppress direct signals for the inspection of PVDF specimen. The active diameter of the receiver (12.5 $\mathrm{mm}$ ) is half the size of the transmitter $(25 \mathrm{~mm})$.

arrives. This signal is typically several magnitudes stronger and can mask all usefull signals. As a result, a transmission inspection setup is used with transmitter, a receiver and sometimes a sound blocker being aligned in one plane along the longitudinal axis of the pipe as shown in Figure 1

Two circular air coupled transducers operating at a nominal frequency of $200 \mathrm{kHz}$ from Ultran (The Ultran Group Inc., State College, PA, USA) are used, one with an active diameter of $25 \mathrm{~mm}$ as transmitter and one with $12.5 \mathrm{~mm}$ diameter as receiver. While the configuration with two smaller (12.5 mm diameter) transducers does not yield a sufficient signal to noise ratio, using a larger (25 mm diameter) transducer to excite a strong Lamb wave and a higher resolution $12.5 \mathrm{~mm}$ diameter receiver to record the signal provides the best setup. Both are mounted on goniometers to adapt to an insonification angle matching the requirements of the specific specimen.

The ultrasonic wave excited by the transducer is a pressure wave in air with a nominal frequency of $200 \mathrm{kHz}$, resulting in a wavelength of $1.7 \mathrm{~mm}$ for a speed of sound in air of $343 \mathrm{~m} / \mathrm{s}$. Both, transmitter and receiver are positioned as close to the specimen surface as possible as indicated with $d_{S}$ in Figure 1 . This also means that the specimen surface is in the near field of the transmitter sound field. Due to the slightly non circular geometry of the pipe, this distance changes from close to $0 \mathrm{~mm}$ to $3 \mathrm{~mm}$ while scanning around a pipe. When the pressure wave hits the surface of the specimen, a Lamb wave in the pipe wall is excited. For maximum 


\begin{tabular}{|l|r|r|}
\hline Specimen & Mode & $\alpha_{T x}, \alpha_{R x}$ \\
\hline PP Pipe & $\mathrm{A}_{0}$ & $19^{\circ}$ \\
PVDF Pipe & $\mathrm{A}_{0}$ & $29^{\circ}$ \\
PMMA Plate & $\mathrm{A}_{0}$ & $19^{\circ}$ \\
PMMA Plate & $\mathrm{S}_{0}$ & $9^{\circ}$ \\
\hline
\end{tabular}

Table 3: Transducer angles for different materials and a frequency of $200 \mathrm{kHz}$.

transition of the wave at the air-structure interface, the projected k-vectors of the wave in the air $\left(k_{\text {air }}\right)$ and in the plate $\left(k_{\text {Lamb }}\right)$ have to match:

$$
k_{a i r} * \sin \left(\alpha_{T x}\right)=k_{L a m b}
$$

From Equation 4 one can calculate the required insonification angle $\alpha_{T x}$. The angles for the measurements are specified in Table 3 for each material and a transducer frequency of $200 \mathrm{kHz}$.

The $\mathrm{A}_{0}$ Lamb wave inside the pipe wall has a wavelength in the range of 3.3-3.5 mm for PVDF and 5.5$6.0 \mathrm{~mm}$ for PP. As Lamb waves feature particle motion over the whole cross-section of the structure, the wave will interact with any defect regardless of its position in depth inside the pipe wall. The propagating Lamb waves also radiate into the surrounding air, allowing a detection with the receiving transducer.

A typical excitation signal for this setup consists of three periods of a sine wave windowed by a Gaussian window. This signal is then amplified up to $400 \mathrm{~V}$ by a Ritec RPR-4000 pulser/receiver (RITEC Inc., Warwick, RI, USA) to drive the transmitter. The use of a continuous excitation signal is not possible because the direct air signal would overlay the Lamb wave signal at the detector.

The received signal is amplified by $40 \mathrm{~dB}$ with a preamplifier and then by another $30 \mathrm{~dB}$ by the amplifier and digitized with $100 \mathrm{MHz}$. The typical range of the signal is $30 \mathrm{mV}_{\mathrm{pp}}$. Up to 256 averages are taken to improve the signal to noise ratio.

\subsection{Lamb wave mode excitation and detection in polymer plates}

The goal of this experiment is to show the dependency of the excitation and detection of different Lamb modes with respect to the transmitter and receiver angle, especially the excitation of a pure $\mathrm{A}_{0}$ Lamb wave. PVDF and PP do not allow this type of investigation due to the high damping factor that does not allow the detection of modes other than the $\mathrm{A}_{0}$ Lamb mode. The experiments are therefore carried out with a PMMA plate. 


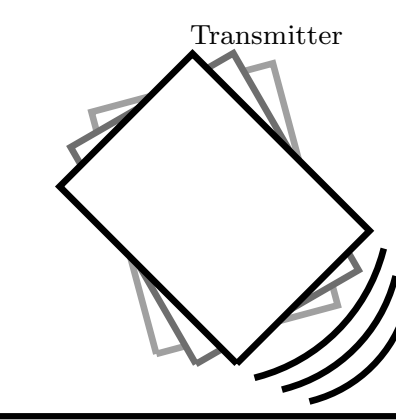

$\sim \sim n$

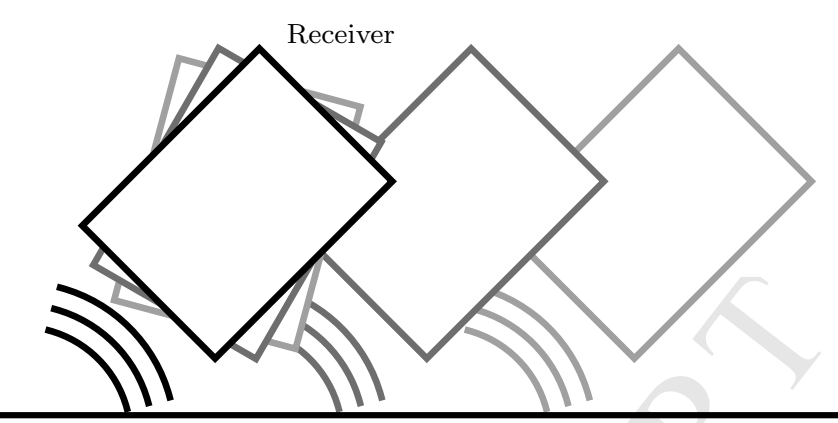

Figure 2: Setup for $\mathrm{A}_{0}$ and $\mathrm{S}_{0}$ Lamb mode excitation and detection. The absoute transmitter angle can be varied over selected angles and the absolute receiver angle can be varied from $0^{\circ}$ to $20^{\circ}$ for each transmitter angle.

The absolute transmitter angle $\alpha_{T x}$ is varied from $0^{\circ}$ to $20^{\circ}$ and receiver angle $\alpha_{R x}$ are varied for every transmitter angle in the same range as shown in Figure 2 and the change of the transmitted signal is investigated.

\subsection{Lamb wave velocity measurement}

To verify the dispersion relations calculated in numerical simulations, phase and group velocity are measured experimentally. Therefore the transmitter-receiver distance is increased by moving the receiver by $10 \mathrm{~mm}$ in steps of $0.1 \mathrm{~mm}$ as shown in Figure 2 With the inspection frequency $f$ being fixed by the transducers, the pipe thickness $d$ is the only parameter that can be varied, resulting in only a small number of measurement points on the dispersion curve.

\subsection{Circumferential pipe inspection}

To identify flaws in the pipe wall, an inspection technique is required that allows to scan along the circumferential pipe direction. This can either be done by turning the pipe and keeping the transducers fixed or by moving the transducers around the pipe. To increase the flexibility with regards to the pipe diameter, a setup with a pipe rotating on wheels is chosen and transmitter and receiver are fixed above the pipe as shown in Figure 1.

PVDF pipes require an additional sound barrier between transmitter and receiver because the low Lamb wave speed results in the arrival of the Lamb wave signal at about the same time as the direct wave through the air which needs to be blocked. Two large rectangular sheets of thick paper with a spacing of $5 \mathrm{~mm}$ to 


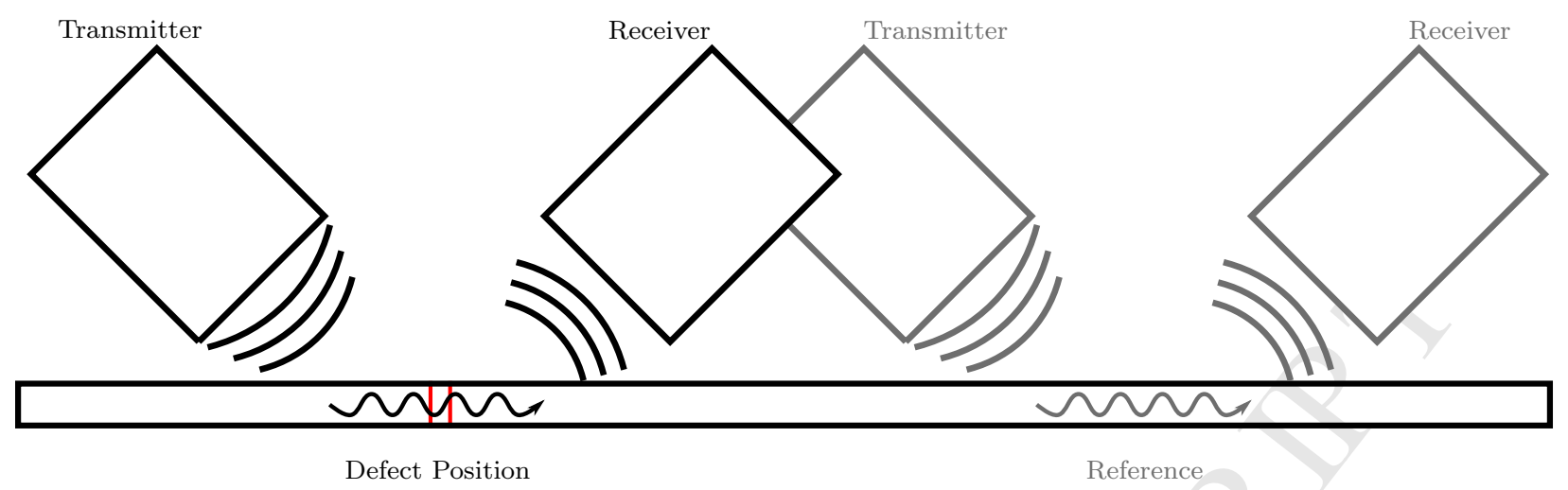

Figure 3: Setup for ACUS reference measurement of pipes. Transmitter and receiver are shifted together by $50 \mathrm{~mm}$ to a flawless position on the pipe.

each other that touch the pipe surface at the topmost position turned out to provide the best results.

\subsection{Setup for reference inspection}

Since geometrical and mechanical inhomogeneities have a big influence on the measured signal from Lamb waves, a reference measurement helps to improve the flaw detection rate. Therefore the transducers are shifted about $\sim 50 \mathrm{~mm}$ along the longitudinal axis of the pipe to an undamaged region, keeping the transducers distance constant. A second scan is performed as reference measurement with exactly the same parameters as shown in Figure 3

\subsection{Signal Analysis}

Figure 9a shows a typical B-Scan for a complete scan around a $110 \mathrm{~mm}$ diameter PVDF pipe and some overlap, leading to more than $400^{\circ}$ scanning range on the $\mathrm{x}$-axis. The amplitude of the $\mathrm{A}_{0}$ Lamb wave is color coded and in the range of $0-30 \mathrm{mV}_{\mathrm{pp}}$. The chosen time frame on the $\mathrm{y}$-axis shows only the Lamb wave. Before the Lamb wave no relevant signal is present and the direct air waves which arrive later have a much higher amplitude.

One can immediately notice the variation of the Lamb wave arrival time with the position. This delay is caused by either local changes in the pipe wall which lead to a change of the Lamb wave velocity or by the out-of-roundness of the pipe that causes a change in the transducer to pipe surface distance and therefore also alters the total travel time of the ultrasonic wave.

An evaluation window is chosen that is shifting with the Lamb wave delay as shown in Figure 9a to avoid 
having the influence of these geometrical effects in the evaluated signal.

The root mean square (RMS) value of the signal within the window is chosen as evaluation function for

\section{Numerical Calculations}

\subsection{Lamb wave propagation simulation}

Proper knowledge of Lamb wave propagation parameters is a prerequisite for the preparation of the experimental setup, especially for the determination of the transducers angles. The Matlab Lamb toolbox [3] a sine wave windowed by a Gaussian window similar to the experimental setup. This wave is propagated forward numerically from the initial position to the defect position with the dispersion parameters of the $\mathrm{A}_{0}$ 
a)

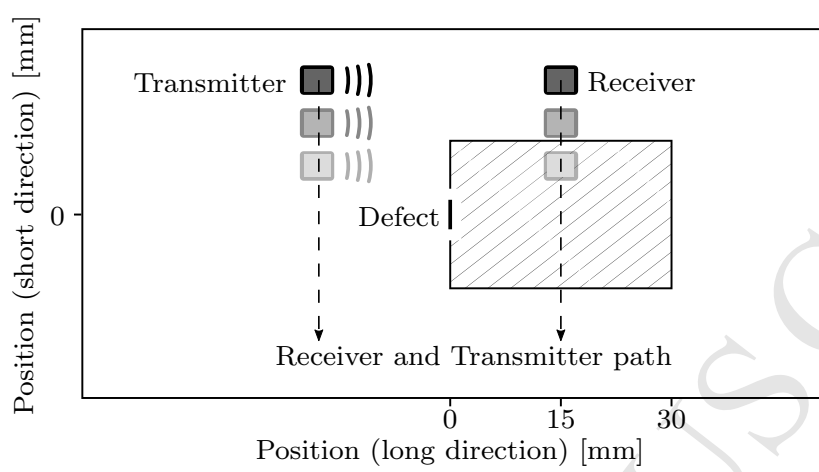

b)

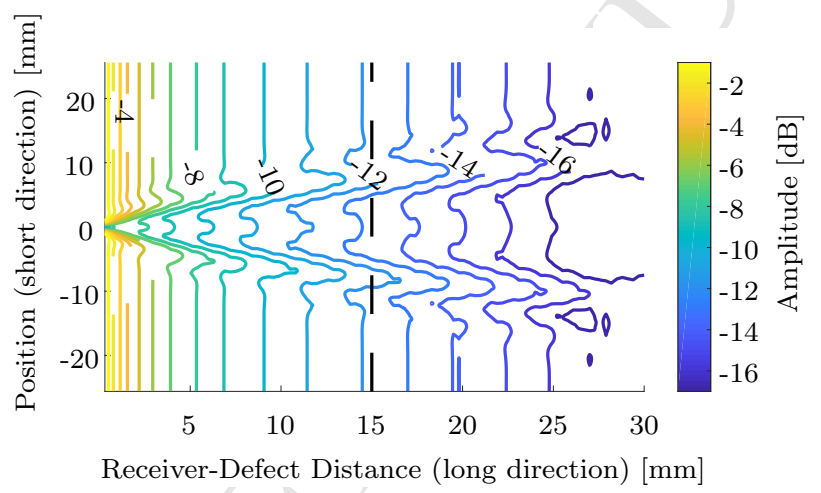

c)

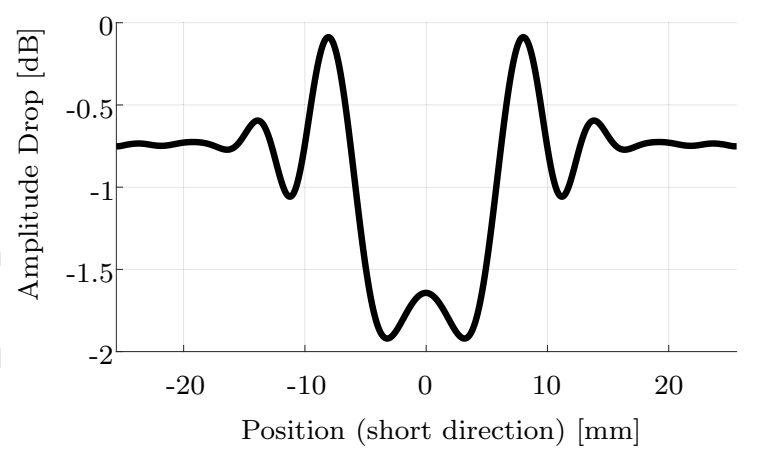

Figure 4: Interaction of Lamb waves in periodic PVDF plates with defects. Diffraction pattern resulting from a plane wave interacting with a $1 \mathrm{~mm}$ plane defect in the center of the specimen at position $(0,0)$ : contour lines $(\mathrm{b})$ of the wave amplitude in an area as shown in a, wave amplitude (c) for a receiver positioned $15 \mathrm{~mm}$ away from the defect as drawn in black in $\mathrm{b}$. 
a)

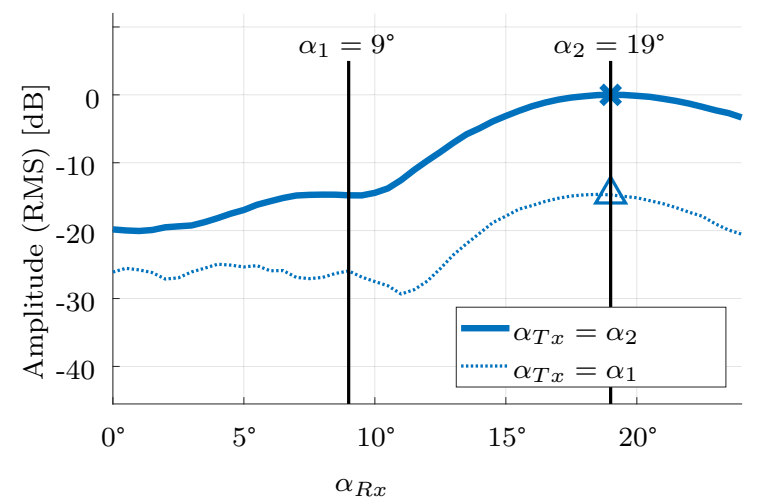

b)

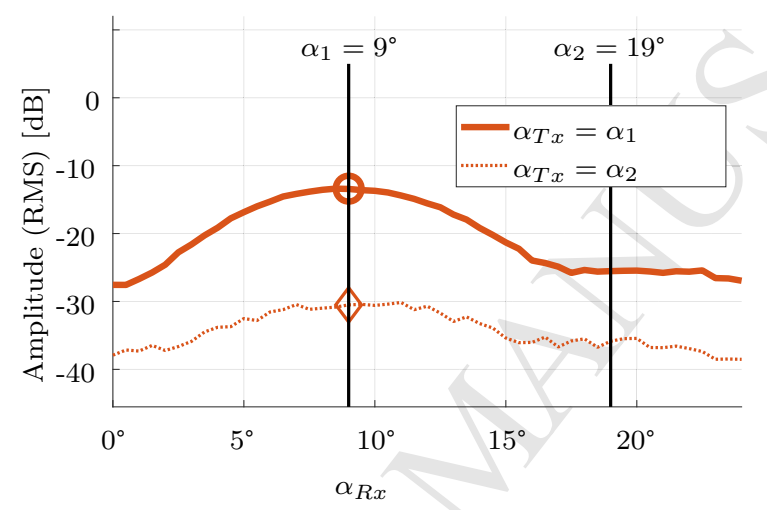

Figure 5: Transducer angle dependency of air coupled $\mathrm{A}_{0}$ (blue, top) and $\mathrm{S}_{0}$ (red, bottom) Lamb wave excitation and detection in PMMA plates.

The $\mathrm{A}_{0}$ Lamb wave mode is depicted for an excitation with a transmitter angle of $19^{\circ}$ (solid blue line) and $9^{\circ}$ (dotted blue line). The receiver signal level is shown for a receiver angle from $0^{\circ}$ to $24^{\circ}$.

The $\mathrm{S}_{0}$ Lamb wave mode is shown for an excitation with a transmitter angle of $9^{\circ}$ (solid red line) and $19^{\circ}$ (dotted red line).

Lamb wave calculated using the Lamb toolbox. The propagation kernel according to Rastogi [14] is used.

At the defect location the wave amplitude is set to zero which is equivalent to no transition of the wave through the defect. The wave is then propagated further and the resulting diffraction pattern in the area behind the defect is shown in Figure 4b. Experimentally, the receiver is not scanning along both, long and short directions of the plate but is only moved along the short direction of the plate at a fixed distance of $15 \mathrm{~mm}$ to the defect. The resulting signal along this path (black in Figure $4 \mathrm{a}$ and $4 \mathrm{~b}$ is shown in Figure $4 \mathrm{c}$ 


\section{Results}

\subsection{Lamb wave mode excitation and detection in thin polymer plates}

For air coupled ultrasonic setups, the transducer angle is of major importance to excite and detect a specific Lamb wave mode. Figure 5 a shows the measured signal amplitude for the $\mathrm{A}_{0}$ Lamb waves for two transmitter angles $\alpha_{1}$ and $\alpha_{2}$ as a function of the receiver angle. Figure $5 \mathrm{~b}$ shows the same results for $\mathrm{S}_{0}$ Lamb waves.

With transmitter and receiver angle matching the optimal $\mathrm{A}_{0}$ angle $\left(\alpha_{T_{x}}=\alpha_{R_{x}}=\alpha_{2}\right)$, the best transmission ratio is archieved (blue cross). This result is expected, as an one sided anti-symmetric excitation prefers the anti-symmetric Lamb modes where the out of plane particle motion is going in the same direction throughout one cross section.

Setting the transmitter and receiver angle to the optimal $\mathrm{S}_{0}$ angle $\left(\alpha_{T_{x}}=\alpha_{R_{x}}=\alpha_{1}\right)$ yields a transmission ratio that is $13 \mathrm{~dB}$ less than the one for the $A_{0}$ mode (red circle).

An $A_{0}$ Lamb wave is also generated as a byproduct when the $S_{0}$ wave is excited with the optimal $S_{0}$ angle $\alpha_{1}$. This is shown as a blue dotted line in Figure $5 \mathrm{a}$ and the signal ampliutde is $15 \mathrm{~dB}$ (blue triangle) lower than for the $\mathrm{A}_{0}$ wave.

Similarly also a $\mathrm{S}_{0}$ Lamb wave can be detected when the $\mathrm{A}_{0}$ wave is excited which is shown as red dotted line in Figure $5 \mathrm{~b}$ This signal is $30 \mathrm{~dB}$ lower than the $\mathrm{A}_{0}$ signal (red diamond).

For the $3 \mathrm{~mm}$ thick PMMA plate used in the experiments, the optimal $\mathrm{S}_{0}$ and $\mathrm{A}_{0}$ transducer angles $\alpha_{1}$ and $\alpha_{2}$ are determined to be $9^{\circ}$ and $19^{\circ}$.

In general the excitation behavior is not very sensitive to small changes of the transducer angle as the peaks in the blue and red curves in Figure 5 are quite broad $\left(-0.2 \mathrm{~dB}\right.$ for a change in angle of $\left.1^{\circ}\right)$.

One can conclude that it is possible to excite an almost pure $A_{0}$ Lamb wave in a plate with air coupled transducers which is important for defect detection in general and the detection of potential $\mathrm{A}_{0}$ to $\mathrm{S}_{0}$ mode conversions at defects.

\subsection{Lamb wave velocity measurements}

Experimentally only the $\mathrm{A}_{0}$ Lamb mode propagation parameters could be measured in PVDF and PP pipes because of the high acoustic damping in these polymers. 
a)

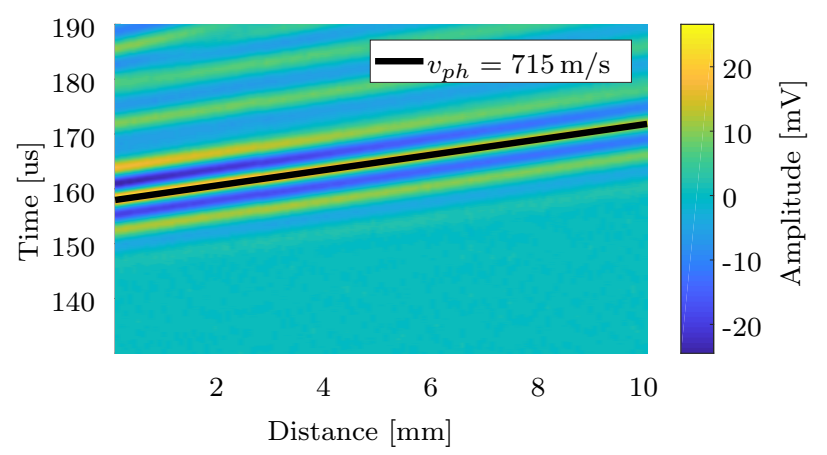

b)

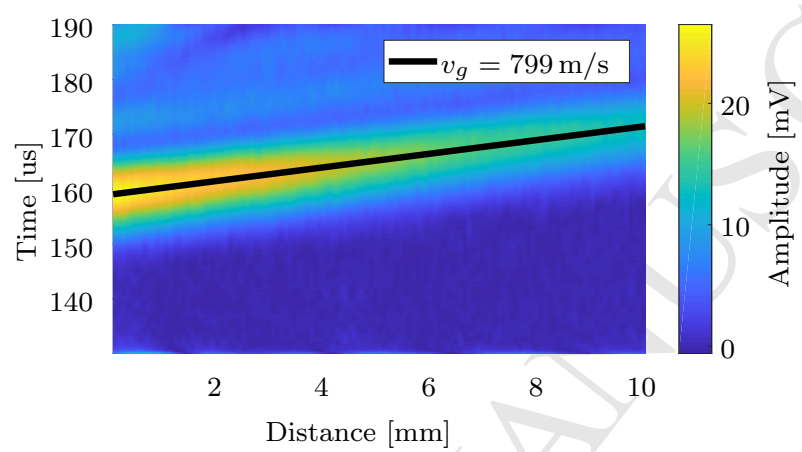

Figure 6: Phase velocity 6a and group velocity 6b measurement for a PVDF pipe with $3.5 \mathrm{~mm}$ thick walls.

The phase velocity is calculated by tracking the maximum of the highest peak with increasing relative transmitter-receiver distance, shown as black line in Figure 6a for a single measurement on a PVDF pipe with $3.5 \mathrm{~mm}$ wall thickness. The group velocity is calculated by tracking the peak of the signal envelope with increasing distance, shown in Figure 6b Several hundred measurements where taken around the circumference of each pipe specimen for averaging.

Figure 7 shows the calculated group and phase velocity of the $\mathrm{A}_{0}, \mathrm{~S}_{0}$ and $\mathrm{A}_{1}$ Lamb wave mode for PVDF as lines and the measured values for the $\mathrm{A}_{0}$ mode at three specific $f \cdot d$ values as crosses. The measured values are all slightly $(4 \%)$ higher than the calculated ones. Figure 8 shows the group and phase velocity for PP, again calculated values as curves and the measurements as crosses. In this case, the simulated results are about $10 \%$ too high. Possible explanations for the differences are discussed in Section 5.1

The results show that the Matlab Lamb toolbox provides a way to get the dispersion relation from material parameters also for polymer pipes and the pipe curvature is negligible in the investigated range. Therefore the measured and the computed values for the wavenumber $k$ and the derived group- and phase velocity can 

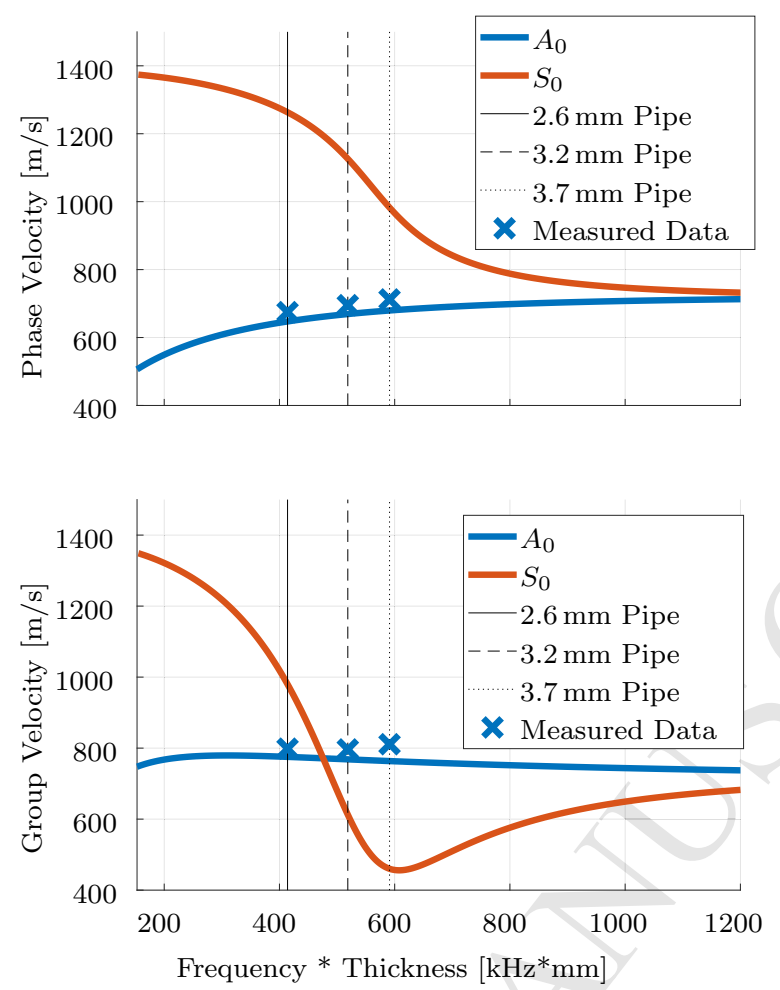

Figure 7: Dispersion Relation for PVDF. Simulated results for plates, measured data for pipes.

be used to prepare the settings of the experiment. With $k$ one can calculate the transmitter and receiver angles from equation 4 . The group velocity helps to determine the distance $d_{\text {Lamb }}$ shown in figure 1 to make sure that the time window is large enough to capture the Lamb waves before the direct air signal arrives. In general, knowing the dispersion relation for the different pulses in the acquired signal allows identifying them as specific Lamb modes.

Additionally, simulations provide a much broader range of frequency values and also include the $\mathrm{S}_{0}$ and other higher Lamb wave modes that may not be possible to be acquired experimentally.

From the measured data, the total attenuation of the $A_{0}$ Lamb wave signal is determined to be in the range of $-5 \mathrm{~dB} / \mathrm{cm}$ for both PVDF and PP pipes. This contains both, inherent damping by the polymers as well as damping of the Lamb wave by radiation into the air and beam divergence.

\subsection{Circumferential pipe inspection}

When scanning the pipe for defects along the circumference, a defect creates a typical signature in the RMS value of the Lamb wave signal. For a receiver diameter of $12.5 \mathrm{~mm}$, this signature has the shape of 

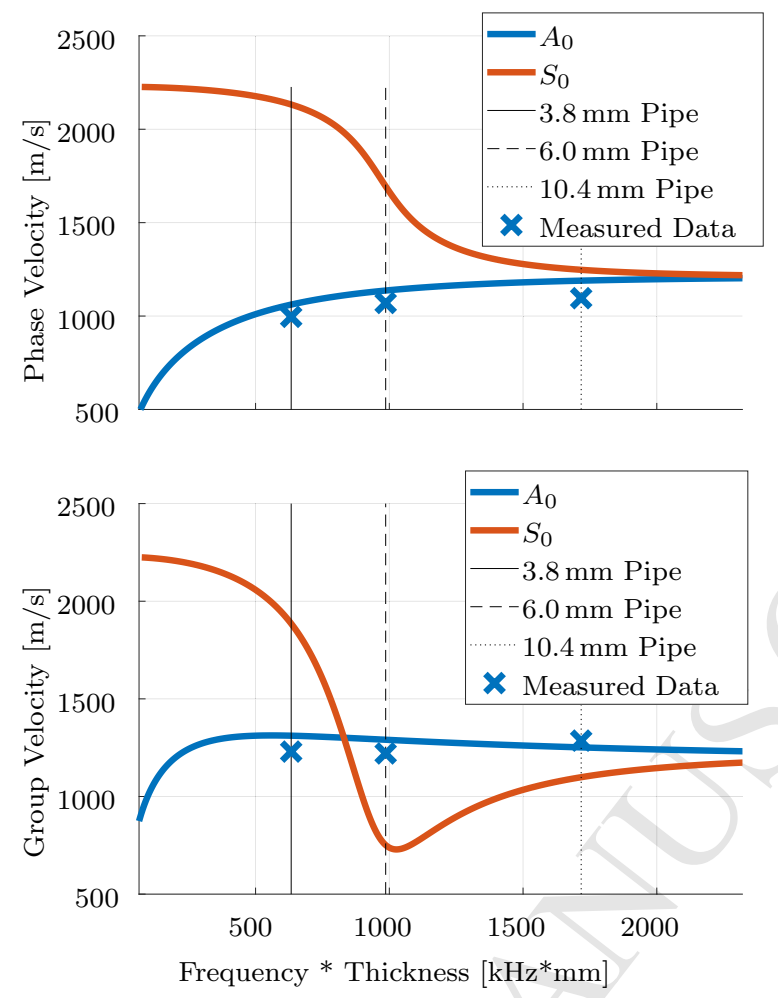

Figure 8: Dispersion Relation for PP. Simulated results for plates, measured data for pipes.

a "W" with two outer peaks and the middle peak at the center of the defect as seen in the blue curve in Figure $9 \mathrm{~b}$ For transducers of diameter $25 \mathrm{~mm}$, the inner peak is missing.

Generally, the noise level in the signal is around $0.5 \mathrm{~dB}$ with the main source being the electrical equipment as motors or power supplies.

The evaluated signal varies for one scan around a specimen by as much as $6 \mathrm{~dB}$ without any defects, mainly due to material inhomogeneities of the specimen and geometrical imperfections, depending heavily on the setup. Major factors are thickness variation, elliptical shape of the pipe and inhomogeneities in the pipe wall from the extrusion process. These effects make the detection of small defects a lot more difficult. While many aspects of the setup geometry may influence the results of air coupled ultrasonic inspection techniques it is difficult to separate them in the chosen setup with non-perfect pipes. Ramadas [15] shows the influence of the transducer misalignment for air coupled transducers in the ideal case of a plate geometry. 
a)

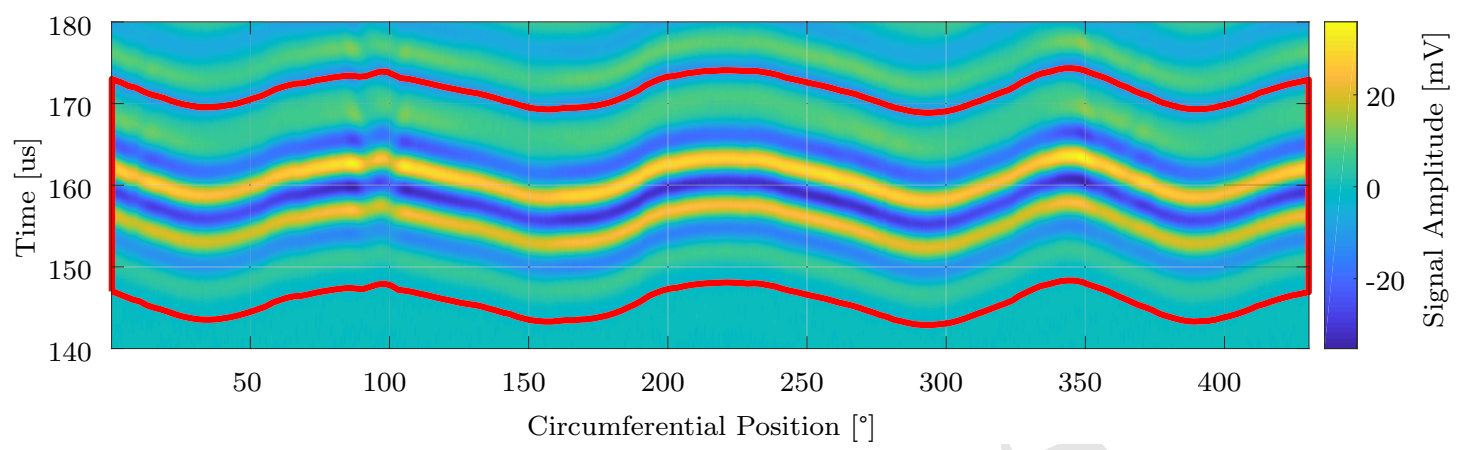

b)

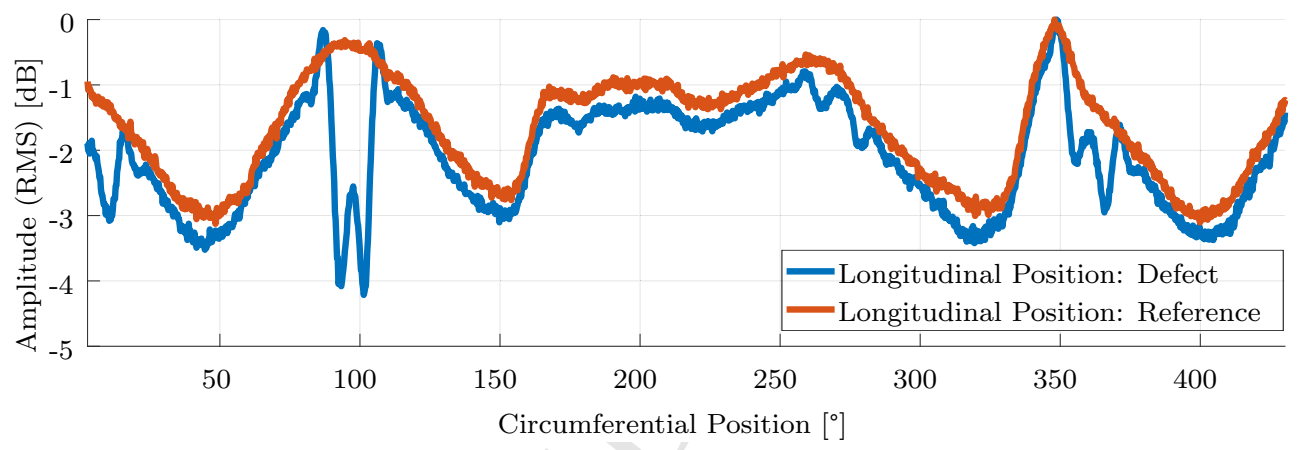

c)

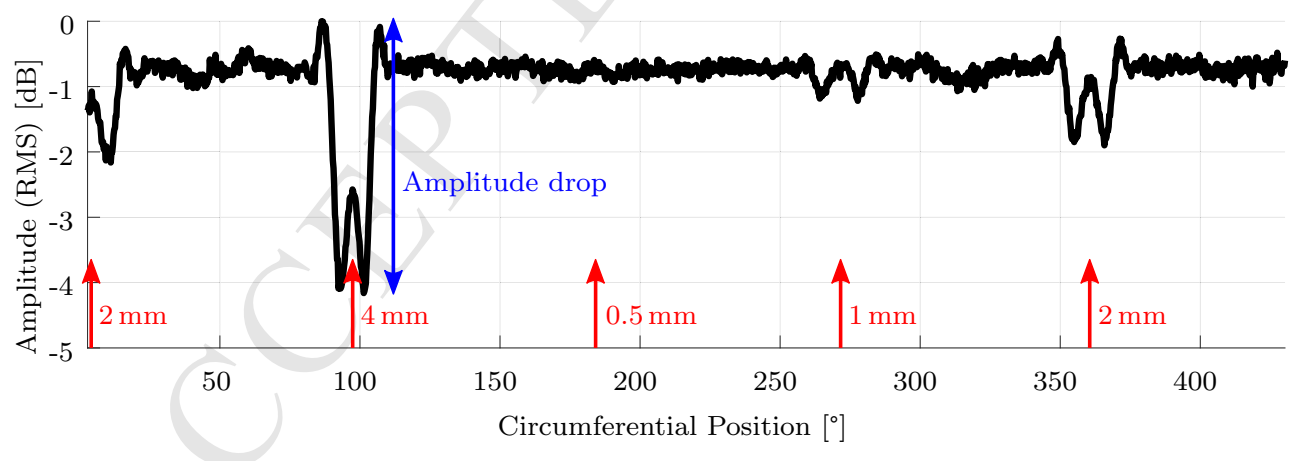

Figure 9: a B-Scan of about $450^{\circ}$ of a $110 \mathrm{~mm}$ PVDF pipe specimen with artificial defects with the evaluation window (red) that is adapting to the Lamb wave delay and allows to evaluate the signal amplitude. Root mean square value (b) of the signal in the evaluation window at the defect position in blue and at the reference position in red. Figure c shows the difference of both evaluations. The impact of the $4 \mathrm{~mm}, 2 \mathrm{~mm}$ and $1 \mathrm{~mm}$ defects is visible and the signal is almost constant otherwise. The probes for the reference measurement were moved in longitudinal direction of the pipe as shown in figure 3 
a)

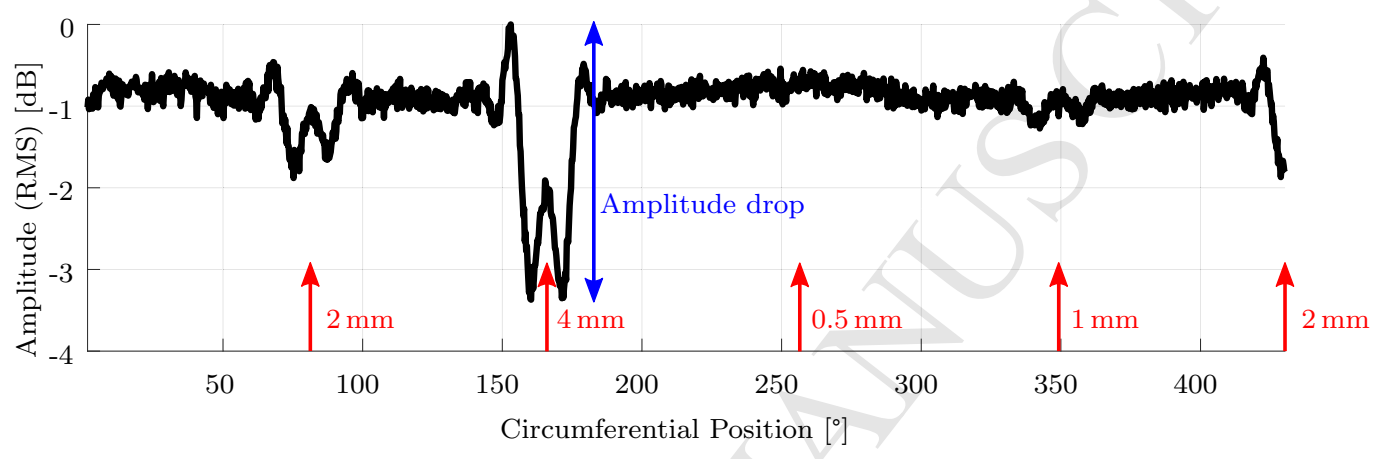

b)

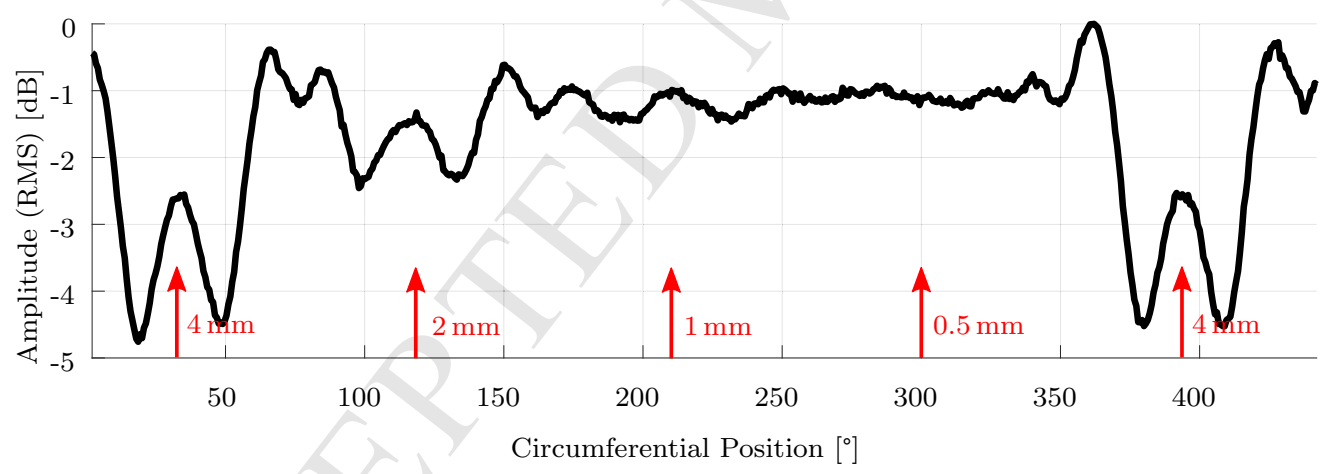

Figure 10: a Results for an inspection of a $110 \mathrm{~mm}$ PP pipe and the amplitude drop for the $4 \mathrm{~mm}$ defect in blue. b Results for a $40 \mathrm{~mm}$ PP pipe for comparison. Note that the circumferential resolution (in $\mathrm{mm}$ ) is the same for both measurements and therefore the number of measurement points is higher for the $110 \mathrm{~mm}$ PP pipe. 
a)

b)
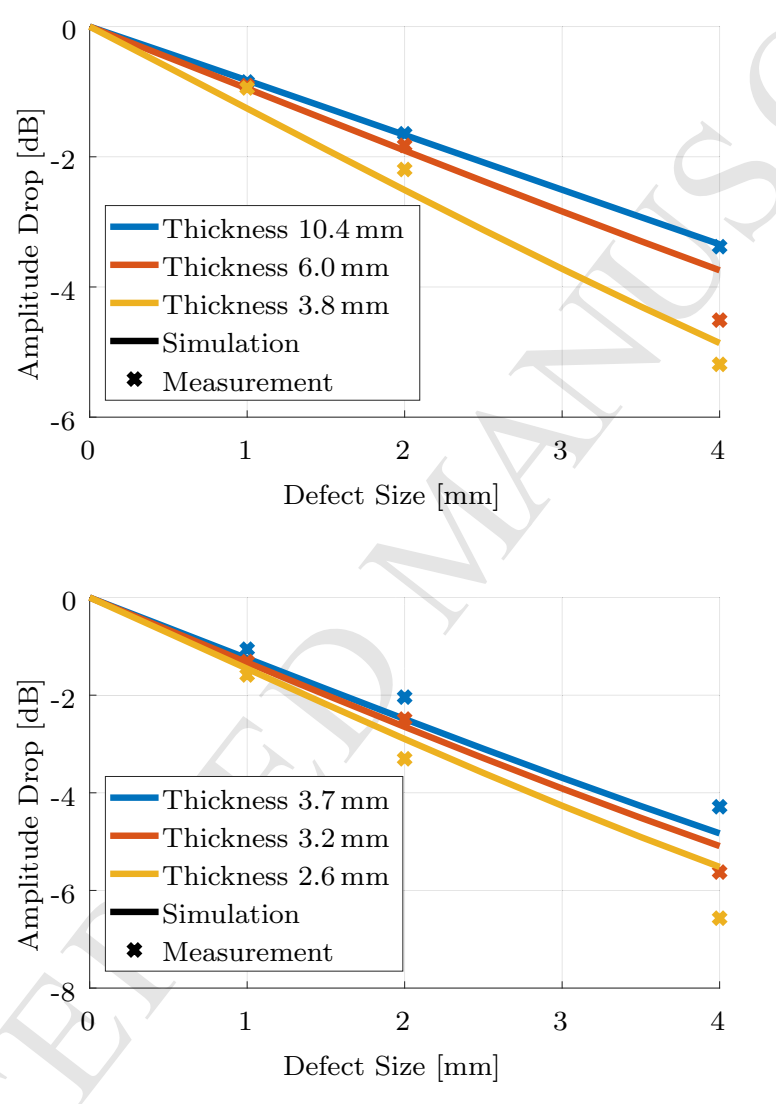

Figure 11: Drop of the signal amplitude for different defect sizes in PP (a) and PVDF (b).

Simulations were carried out for defects from $0 \mathrm{~mm}$ to $4 \mathrm{~mm}$ in small steps and are shown as connected lines. Measurements were conducted with $1 \mathrm{~mm}, 2 \mathrm{~mm}$ and $4 \mathrm{~mm}$ defects and are shown with crosses. 


\subsection{Detection limit improvement with reference measurement}

PP but the amplitude drop is in general a bit higher.

\section{Discussion}

Inspections of PVDF pipes were possible only with the help of a sound blocker that prevents direct transmission of the ultrasonic wave through the air. The additional time frame that is gained with a sound

blocker is only a few microseconds, afterwards sound waves arrive at the transducer that are reflected at various other surfaces, e.g. the pipe wall. Since the sound blocker is touching a part of the pipe surface that is examined, the Lamb waves might be damped and it cannot be excluded that mode conversions occur. The transducers are mounted as close to the specimen surface as possible as indicated with d in Figure 1 This also means that the natural focal point ( $47 \mathrm{~mm}$ for transducers with $25 \mathrm{~mm}$ active diameter) at the 

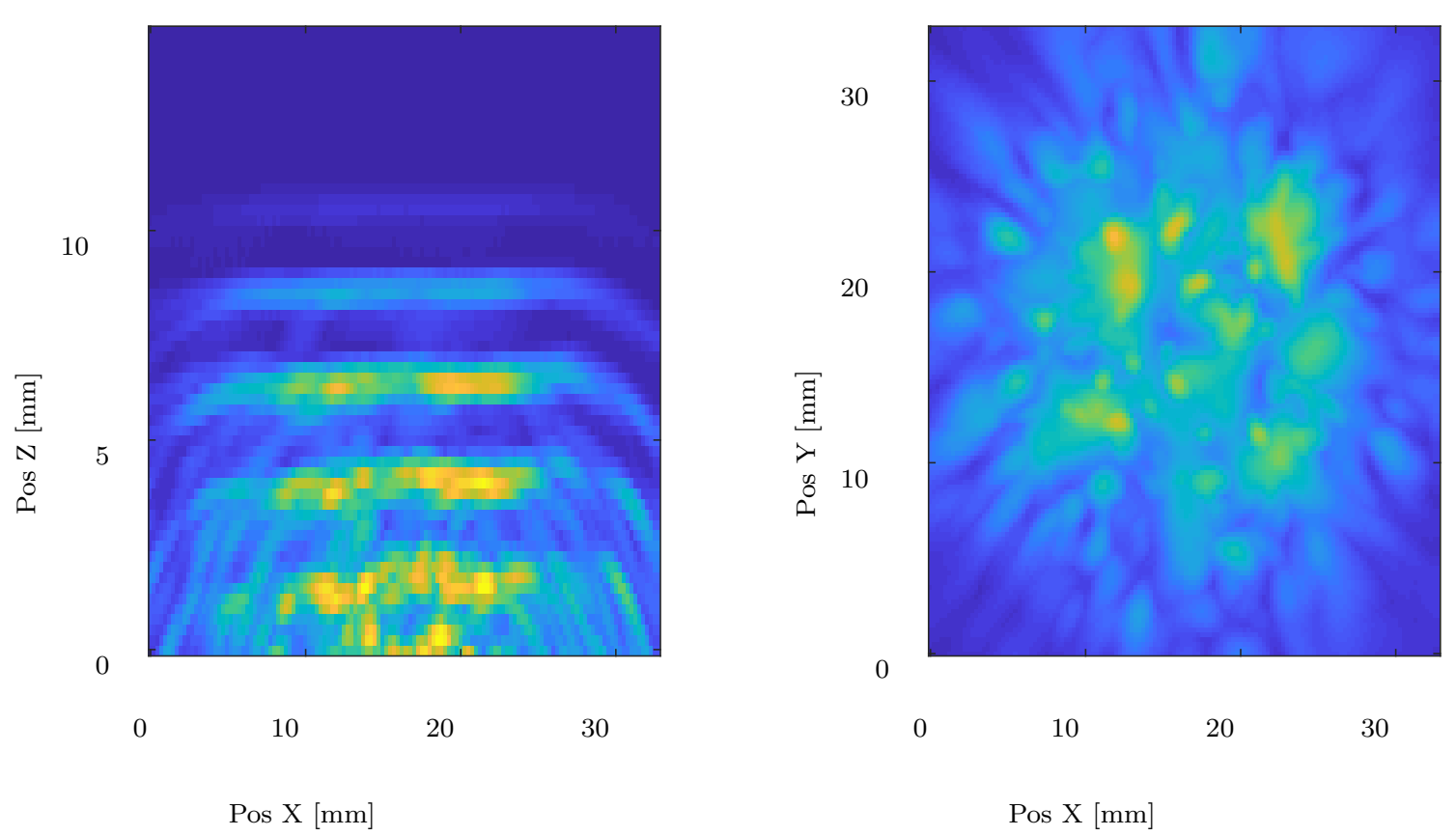

Figure 12: Measured sound field of a $25 \mathrm{~mm}$ Ultran ultrasonic transducer in lateral (left) and frontal (right) view.

\subsection{Numerical wave propagation}

The simulations for the effect of defects give a good prediction on the signal shape and also show the same trend as the measurements. However, as expected for a simplified model, the absolute values differ slightly. Several effects of the propagation model may contribute to this effect. First of all, the geometry is 


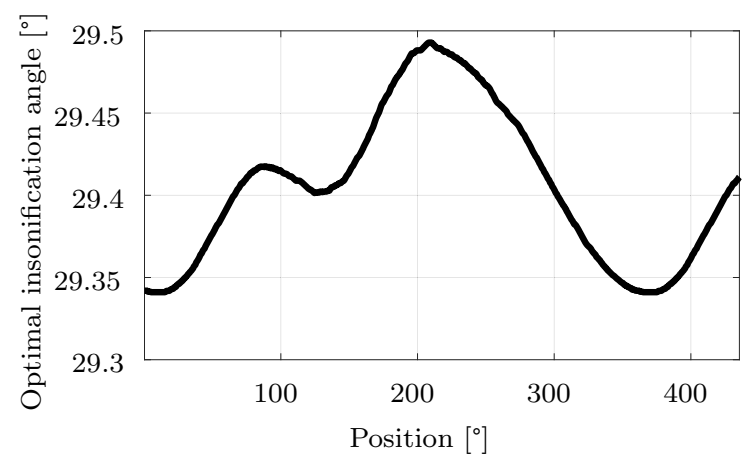

Figure 13: Pipe optimal insonification angle for $A_{0}$ Lamb mode in a $110 \mathrm{~mm}$ PVDF pipe calculated from the measured real wall thickness.

modelled as a plate with a plane wave propagating, whereas in reality the wave is originating from the spot assuming a constant density distribution over the pipe circumference, the optimal insonification angle for 
the $\mathrm{A}_{0}$ Lamb waves can be calculated from the data gained from the Lamb toolbox simulations. As it can be seen in Figure 13 , the total variation of the optimal angle for a total thickness variation of $0.25 \mathrm{~mm}$ is less wave transmission level as shown in Figure 5 for PMMA, such small angle variations should not have a major impact on the measured signal.

\section{Conclusion}

It is possible to detect artificial defects using air coupled ultrasonic inspection in thin polymer pipe walls.

\section{Acknowledgements}

This work was supported by the Commission for Technology and Innovation (CTI) located at the Federal Department of Economic Affairs (FDEA), Switzerland (project no. 17103.1 PFIW-IW). 
[10] M. J. Padiyar, K. Balasubramaniam, Lamb wave-based air-coupled ultrasonic inspection technique for filament-wound composite pipes, Insight - Non-Destructive Testing and Condition Monitoring 56 (4) (2014) 195-202. doi:10.1784/insi.2014.56.4.195

[1] M. Castaings, P. Cawley, The generation, propagation, and detection of Lamb waves in plates using air-coupled ultrasonic transducers, The Journal of the Acoustical Society of America 100 (5) (1996) 3070-3077. doi:10.1121/1.417193.

[2] J. L. Rose, Ultrasonic guided waves in solid media, Cambridge University Press, 2014.

[3] J. L. Prego Borges, Lamb: a simulation tool for air-coupled Lamb wave based ultrasonic NDE systems, PhD Thesis, Polytechnic University of Catalonia, Barcelona, Spain (2010)

[4] B. Hartmann, J. Jarzynski, Immersion apparatus for ultrasonic measurements in polymers, The Journal of the Acoustical Society of America 56 (5) (1974) 1469-1477, doi:10.1121/1.1903466.

[5] D. Christman, Dynamic properties of PMMA, General Motors Technical Center, Rep. AD-743547 (1972)

[6] T. Leutenegger, J. Dual, Non-destructive testing of tubes using a time reverse numerical simulation (TRNS) method, Ultrasonics 41 (10) (2004) 811-822. doi:10.1016/j.ultras.2003.12.046.

[7] C.-T. Ng, M. Veidt, Scattering of the fundamental anti-symmetric lamb wave at delaminations in composite laminates, The Journal of the Acoustical Society of America 129 (3) (2011) 1288-1296. doi:10.1121/1.3533741.

[8] J. McKeon, M. Hinders, Lamb wave scattering from a through hole, Journal of Sound and Vibration 224 (5) (1999) 843-862.

[9] P. Fromme, M. B. Sayir, Measurement of the scattering of a Lamb wave by a through hole in a plate, The Journal of the Acoustical Society of America 111 (3) (2002) 1165-1170. doi:10.1121/1.1448338 
[11] F. Benmeddour, S. Grondel, J. Assaad, E. Moulin, Study of the fundamental lamb modes interaction with asymmetrical discontinuities, NDT \& e International 41 (5) (2008) 330-340.

345

[14] P. K. Rastogi, E. Hack, Optical methods for solid mechanics: a full-field approach, John Wiley \& Sons, 2012.

[15] C. Ramadas, A. Hood, I. Khan, K. Balasubramaniam, Effect of misalignment of air-coupled probes on ultras.2014.02.014.

[16] J. Neuenschwander, R. Furrer, A. Roemmeler, Application of air-coupled ultrasonics for the characterization of polymer and polymer-matrix composite samples, Polymer Testing 56 (2016) 379-386. 


\section{CRediT author statement}

Antonio de Agostini: Project Administration, Funding Acquisition. Jürg Dual: Writing - Review \& Editing. Roman Furrer: Resources. Bastian Lübke: Supervision. Jürg Neuenschwander: Conceptualization, Project Administration, Funding Acquisition, Writing - Review \& Editing. Arno Römmeler: Investigation, Writing - Original Draft, Visualization. Urs Sennhauser: Supervision. Jörg Wermelinger: Resources. Peter Zolliker: Software, Writing - Review \& Editing. 